\title{
A proof-of-concept analysis of carbohydrate-deficient transferrin by imaged capillary isoelectric focusing and in-capillary immunodetection
}

$\overline{\text { Jiaqi Wu*,1, Charles H Haitjema', Christopher D Heger' \& Annegret Boge' }}$

\begin{abstract}
Carbohydrate-deficient transferrin (CDT) is a reliable biomarker for chronic alcohol abuse. We developed a method for CDT analysis by capillary isoelectric focusing, followed by immunodetection directly in the capillary, in an automated fashion and on a single platform (Peggy Sue ${ }^{\mathrm{TM}}$; ProteinSimple, CA, USA). Transferrin glycoforms in serum samples, including disialo-transferrin, were separated and their apparent isoelectric points and relative percentages were determined. The relative CDT values (percent of total transferrin) matched expected values for both healthy and alcoholic samples. Because the method leveraged the sensitivity of an immunoassay, CDT was measured when serum samples were diluted up to 1200 -fold, reducing the volume of serum required. Finally, the process is fully automated, with up to 96 samples analyzed per batch.
\end{abstract}

\section{METHOD SUMMARY}

Carbohydrate-deficient transferrin is a common biomarker for diagnosing chronic alcohol abuse. Here, we developed a technique for the detection of carbohydrate-deficient transferrin by isoelectric focusing followed seamlessly by immunodetection directly in the capillary. The method offers several advantages over existing techniques, including small sample size $(15 \mu \mathrm{l})$, specificity and automation, while also providing a complete charge-based separation profile of transferrin.

\section{KEYWORDS}

carbohydrate-deficient transferrin - imaged capillary isoelectric focusing - immunodetection • Simple Western

'ProteinSimple, a Bio-TechneBrand, 3001 Orchard Parkway, San Jose, CA 95134, USA; *Author for correspondence: jiaqi.wu@bio-techne.com

BioTechniques 68: 85-90 (February 2020) 10.2144/btn-2019-0058

\section{GRAPHICAL ABSTRACT}

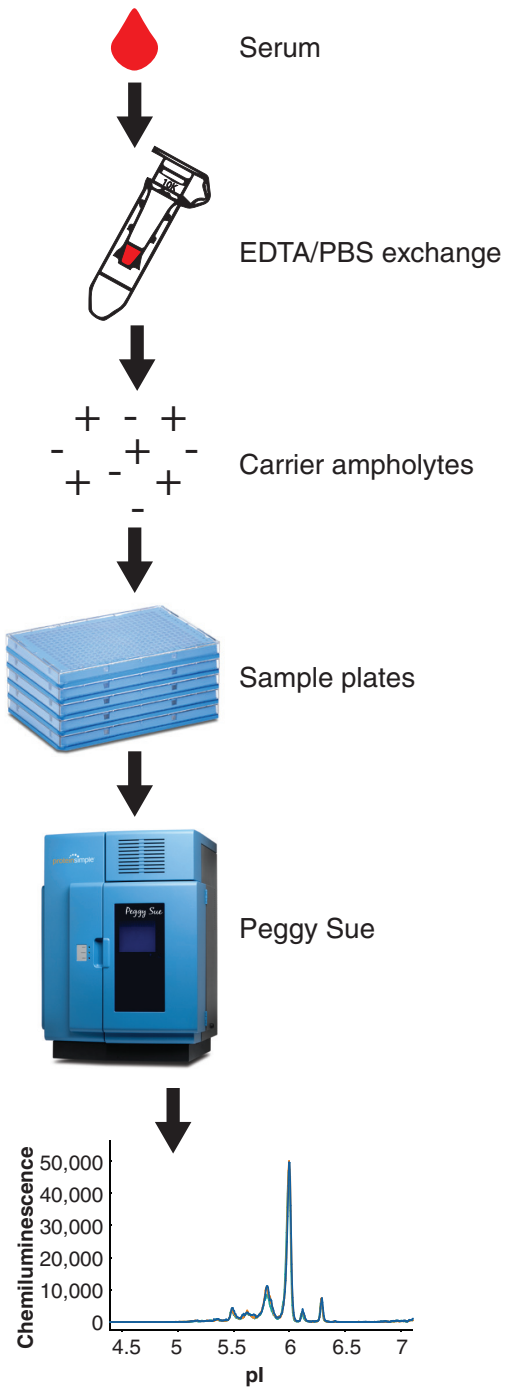

Transferrin (Tf) is an important iron-transporting protein in human serum. With two available $N$-linked glycosylation sites, several glycoforms of Tf exist, depending on the attachment of zero-two $\mathrm{N}$-glycans. These $\mathrm{N}$-glycans display a varying number of terminal sialic acid residues, and this number is used to name each glycoform. For example, in healthy individuals, the major Tf glycoform contains four sialic acid residues (tetrasialo-Tf) with minor glycoforms of disialo-Tf (two sialic acid residues), trisialo-Tf (three sialic acid residues), pentasialo-Tf (five sialic acid residues) and hexasialo-Tf (six sialic acid residues) [1]. By contrast, chronic alcohol abusers, defined as those that consume more than $50 \mathrm{~g}$ of alcohol a day for greater than seven consecutive days, typically have elevated levels of carbohydrate-deficient Tf (CDT), which refers collectively to asialo-Tf (nonsialylated) and disialo-Tf [2]. This makes CDT a reliable and specific biomarker to identify chronic alcohol abuse. In addition to Tf glycoforms, other isoforms of $\mathrm{Tf}$ exist owing to genetic polymorphisms caused by one or more amino acid substitutions to the primary structure of the protein. Therefore, the monitoring of $\mathrm{Tf}$ glycoforms and genetic isoforms is important in clinical and forensic analysis of chronic alcohol abuse, the investigation of genetic variants and the assessment of congenital disorders of glycosylation [1,2].

Tf glycoforms confer different isoelectric point $(\mathrm{pl})$ values to the molecule. Specifically, Tf glycoforms with many sialic acid residues have lower pl values, and Tf glycoforms with few sialic acid residues have higher pl values. Because CDT is deficient in sialic acid residues, it has a higher overall $\mathrm{pl}$ than other glycoforms, and this property is leveraged to separate CDT from other glycoforms in charge-based separation methods. Early isoelectric focusing (IEF) methods relied on labor-intensive slab gels, followed by immunodetection that are only semiquantitative [2]. Other methods for CDT analysis have been reported, including those based on capillary zone electrophoresis [3] and HPLC [4], the latter of which is currently the IFCC reference method for CDT measurement. However, the detection modes in these methods are typically based on UV/visible absorption or native fluorescence [5], and, therefore, have limited sensi- 
tivity. Recently, the sensitivity was improved by harnessing the ability of $\mathrm{Tf}$ to bind $\mathrm{Tb}^{3+}$, which greatly enhances the intrinsic fluorescence of $\mathrm{Tf}$, resulting in a relevant increase in analytical sensitivity by a factor of approximately 3 [6]. A CDT-targeting antibody can be used to leverage the high sensitivity of immunoassay [7]. However, a drawback of this method is the antibody does not distinguish disialo-Tf and asialo-Tf from monosialo-Tf, which is not considered CDT [7].

Capillary IEF (CIEF) can be used to analyze Tf, and it is both quantitative and automated [8]. As its name suggests, cIEF brings IEF inside a capillary to separate molecules based on their pl. Because cIEF uses a capillary instead of a slab gel, as in conventional IEF, the method has improved resolution, speed and sensitivity. Although this method has the advantages of automation and quantitation, it requires a mobilization step to move the focused material through the detector that is located at one end of the capillary [8]. This mobilization step takes longer than the focusing step itself, and it can alter the separation resolution and impact the reproducibility. These mobilization-related issues prevented CIEF from being widely used in bioanalytical chemistry. Furthermore, immunoextraction of $\mathrm{Tf}$ from the serum is typically required before analysis by $\mathrm{CIEF}[8]$.

The development of imaged cIEF (icIEF) eliminated the need for a mobilization step, and thus alleviated its associated problems, making it an easier way to perform IEF in a capillary [9]. Instead of mobilizing the focused material through a detector located at one end of the capillary, the entire capillary is monitored throughout the focusing process. This in turn facilitates faster method development, and enhances reproducibility, robustness and speed. The technology advanced the potential of capillary-based IEF technology, and it became the gold standard method for IEF [10]. Tf glycoforms in human serum samples were previously analyzed by icIEF and required little sample pretreatment [11]. The method was precise and could determine the $\mathrm{pl}$ of each glycoform within $0.03 \mathrm{pH}$ units [11]. However, this iclEF method still has limitations in sensitivity, since its detector is based on UV absorption and not immunodetection.
In recent years, a new IEF method has emerged that combines the principles of iclEF with immunodetection [12]. Following IEF, proteins are immobilized to the capillary wall by a UV-catalyzed chemical reaction. Then, antibodies are used for sensitive and specific immunodetection of charge variants. This assay, also called Simple Western ${ }^{\text {TM }}$ Charge, can similarly be applied to proteins separated by size, called Simple Western Size [13]. Simple Western Charge was employed to rapidly characterize sialylation patterns without requiring protein purification [14]. In this study, we introduce a proofof-concept for CDT analysis that combines the charge-based separation of iclEF with the sensitivity of immunodetection, both of which are performed seamlessly back to back on a single Simple Western platform (Peggy Sue $^{T M}$ from ProteinSimple, CA, USA). Serum samples can be diluted directly in sample buffer with only minor pretreatment to remove iron ions. The sensitivity of this method eliminated the need for immunopurification of Tf from human serum samples that is typically required for CIEF [8]. Furthermore, the method enables the analysis of up to 96 samples in a fully automated fashion overnight $(<15 \mathrm{~h})$. Finally, we compared the results from this assay with traditional icIEF by native fluorescence detection (Maurice ${ }^{\text {TM }}$ from ProteinSimple).

\section{MATERIALS \& METHODS} Instruments

icIEF with immunodetection method was run on the Peggy Sue Simple Western instrument (ProteinSimple). The capillary is $5 \mathrm{~cm}$ long, $100 \mu \mathrm{m}$ internal diameter and holds $400 \mathrm{nl}$ of solution. Because samples are immobilized on the capillary wall prior to immunodetection, capillaries are single use. Traditional iclEF experiments were run on ProteinSimple's Maurice instrument with fluorescence detection $(280 \mathrm{~nm}$ excitation, 320-450 nm emission). The voltage settings were $1500 \mathrm{~V}$ for prefocusing and $3000 \mathrm{~V}$ for focusing.

\section{Reagents \& devices}

Serum sample controls (Normal Control and High CDT Control) were purchased from Sebia (GA, USA). icIEF sample buffer (Premix G2 Ampholyte-Free pH Gradient, PN 040-967), 1\% methylcellulose solution (PN 101876), antibody diluent (antibody diluent 2 ,
PN 042-203), anti-mouse secondary antibody (ready-to-use format, PN 042-205), pl standard ladder 1 (containing $5 \mathrm{pl} \mathrm{markers,}$ ranging from 4.0 to 7.3 , PN 040-644), luminol solution (luminol-S, PN 043-311), column wash buffer (PN 041-108), anolyte (PN 040-337), catholyte (PN 040-338) and peroxide solution (PN 043-379) used for the Peggy Sue system were products of ProteinSimple. Anti-human Tf mouse monoclonal antibody (PN MAB5746), purified human IgG (PN 1-001-A) and purified human Tf (PN 2914-HT) were purchased from R\&D Systems (MN, USA). Phosphate-buffered saline (PBS) solution, urea powder, sialidase (PN 10269611001), sodium citrate dihydrate and citric acid were purchased from Sigma (MS, USA). Pharmalytes 5-8 (PN 17045301) and Pharmalytes 3-10 (PN 17045601) were products of GE Healthcare (IL, USA). The Amicon ${ }^{\circledR}$ Ultra -0.5 -ml Centrifugal Filter 10 K NMWL (PN UFC501096) was used as the centrifuging protein dialysis tube and was purchased from Sigma.

\section{Sample treatment \& preparation for analysis}

For analysis on both Peggy Sue and Maurice, iron ions in Tf were removed using EDTA ( 0.17 $M$ EDTA solution in $0.2 \mathrm{M}$ citrate buffer, $\mathrm{pH}$ 4.0). First, $15 \mu \mathrm{l}$ of each serum sample was treated twice with $150 \mu \mathrm{l}$ EDTA solution using the Amicon Ultra Centrifugal Filter by spinning at $14,000 \mathrm{rpm}$ at $4^{\circ} \mathrm{C}$ down to a final volume of $30 \mu \mathrm{l}$. Then, the sample was buffer exchanged into $0.5 \times$ PBS by equilibrating twice with $150 \mu \mathrm{l} 0.5 \times$ PBS using the same Amicon Ultra Centrifugal Filter and spinning at $14,000 \mathrm{rpm}$ at $4^{\circ} \mathrm{C}$ to a final volume of approximately $30 \mu \mathrm{l}$. All samples were treated and prepared fresh on the same day as the analysis. Samples were stored on ice until use.

For analysis on Peggy Sue, the purified Tf and CDT serum samples were diluted directly into the final IEF sample solution containing Premix G2 ampholyte-free premix, 3\% Pharmalyte $5-8,1 \%$ Pharmalyte $3-10$ and pl ladder solution. The serum samples were typically diluted into the IEF buffer by a dilution factor of $1 / 600$, which was determined to be in the linear range of detection from a $2 \times$ serial dilution series from $1 / 50$ to $1 / 1600$. The final concentration of purified Tf was $5 \mu \mathrm{g} / \mathrm{ml}$.

For analysis on Maurice, the purified Tf and CDT samples were diluted directly 
into the IEF buffer containing $8 \mathrm{M}$ urea, 3\% Pharmalyte 5-8, 1\% Pharmalyte 3-10, 0.35\% methylcellulose solution and pl markers 5.85 and 7.05 . The final concentration of purified Tf was $0.16 \mathrm{mg} / \mathrm{ml}$, and the serum samples were diluted into the IEF buffer by a dilution factor of $1 / 25$.

\section{Antibody preparation}

The Tf primary antibody was diluted $1 / 200$ in antibody diluent. For the secondary antibody, human IgG $(60 \mu \mathrm{g} / \mathrm{ml})$ was spiked into the ready-to-use secondary antibody solution to reduce nonspecific recognition of serum proteins by the secondary antibody.

\section{Sample run \& data processing}

Sample solutions and antibody solutions were loaded onto a 384-well plate. The plate was centrifuged at $2500 \mathrm{rpm}$ for $10 \mathrm{~min}$ at $4^{\circ} \mathrm{C}$ and then inserted into the Peggy Sue, which keeps the sample plate at approximately $4^{\circ} \mathrm{C}$. The IEF time was set to $50 \mathrm{~min}$ using a constant electric power setting of 21,000 microwatts. On Peggy Sue, the incubation times for the primary antibody and secondary antibody were 60 and $30 \mathrm{~min}$, respectively. For Maurice iclEF runs, the focusing time was $1 \mathrm{~min}$ at $1.5 \mathrm{kV}$ followed by $14 \mathrm{~min}$ at $3 \mathrm{kV}$. All data were processed using Compass software.

\section{RESULTS \& DISCUSSION \\ Workflow overview}

The workflow for CDT analysis developed here is shown in Figure 1. First, $15-\mu$ l serum samples are treated with EDTA to remove iron, and then resuspended in PBS. Then, samples are diluted 600 -fold in the IEF sample solution containing carrier ampholytes, urea and pl markers. Finally, samples are transferred to a 384-well Peggy Sue sample plate and loaded on Peggy Sue. A protocol is included along with this paper that can be followed to analyze any human serum sample by this method.

Upon starting the instrument, samples are automatically loaded into capillaries in which IEF is performed. After focusing, the separated proteins are immobilized onto the capillary walls by a UV-catalyzed reaction. Then, the capillaries are washed to remove the IEF buffer solution, and a primary antibody specific for Tf is applied into the capillaries, followed by an HRP-conjugated secondary antibody. Finally, a luminol-

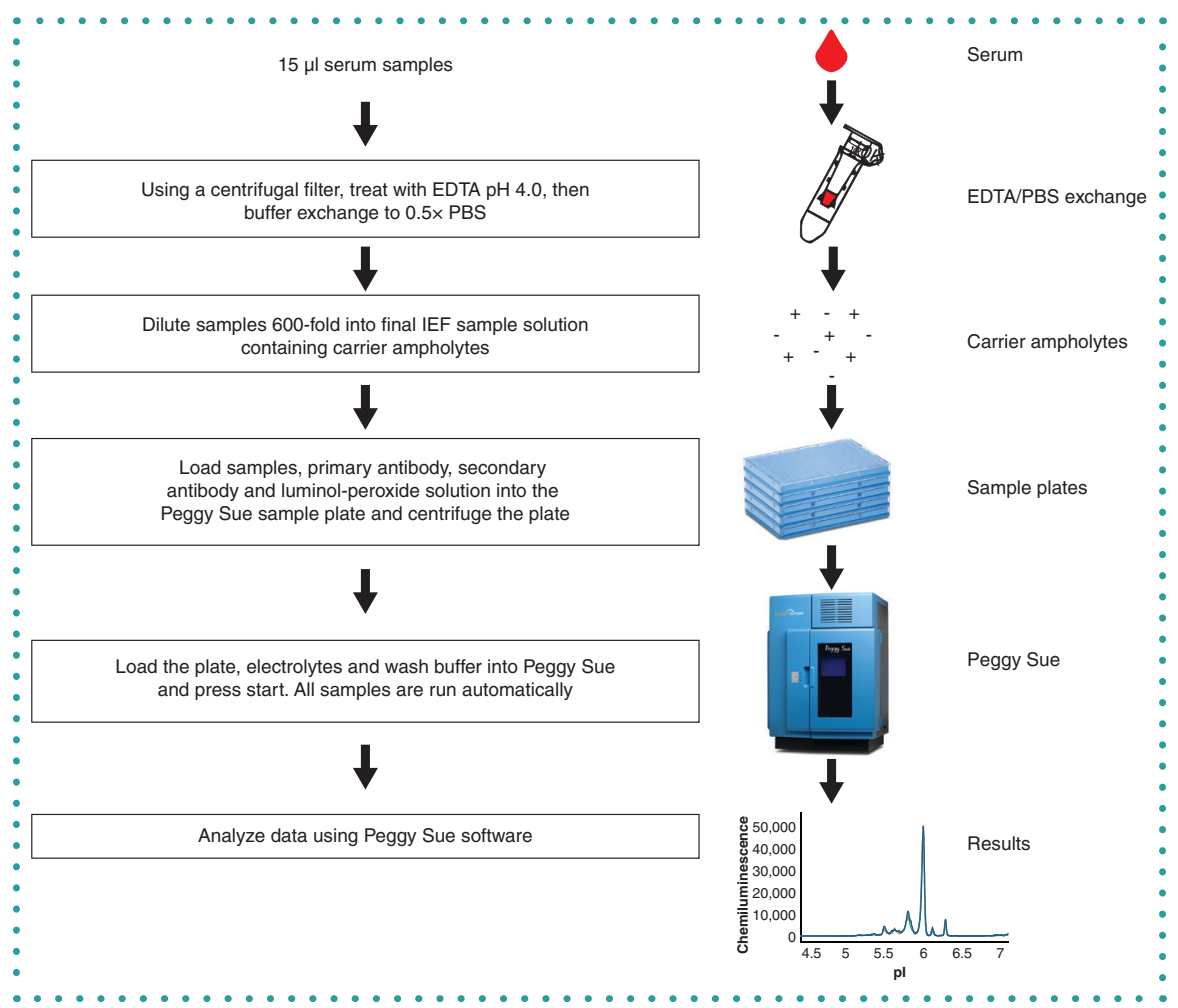

Figure 1. Workflow of carbohydrate-deficient transferrin analysis by Simple Western Charge. IEF: Early isoelectric focusing.

peroxide solution is applied for chemiluminescence imaging of the whole capillary. More information on the principles of this assay may be found in a similar study that characterized protein sialylation patterns [14]. Following sample preparation,

all steps are automatically performed on the Peggy Sue instrument without the need for analyst supervision. On Peggy Sue, 12 samples are focused simultaneously in each cycle, and cycles are executed in parallel and overlap with one another. Therefore, up

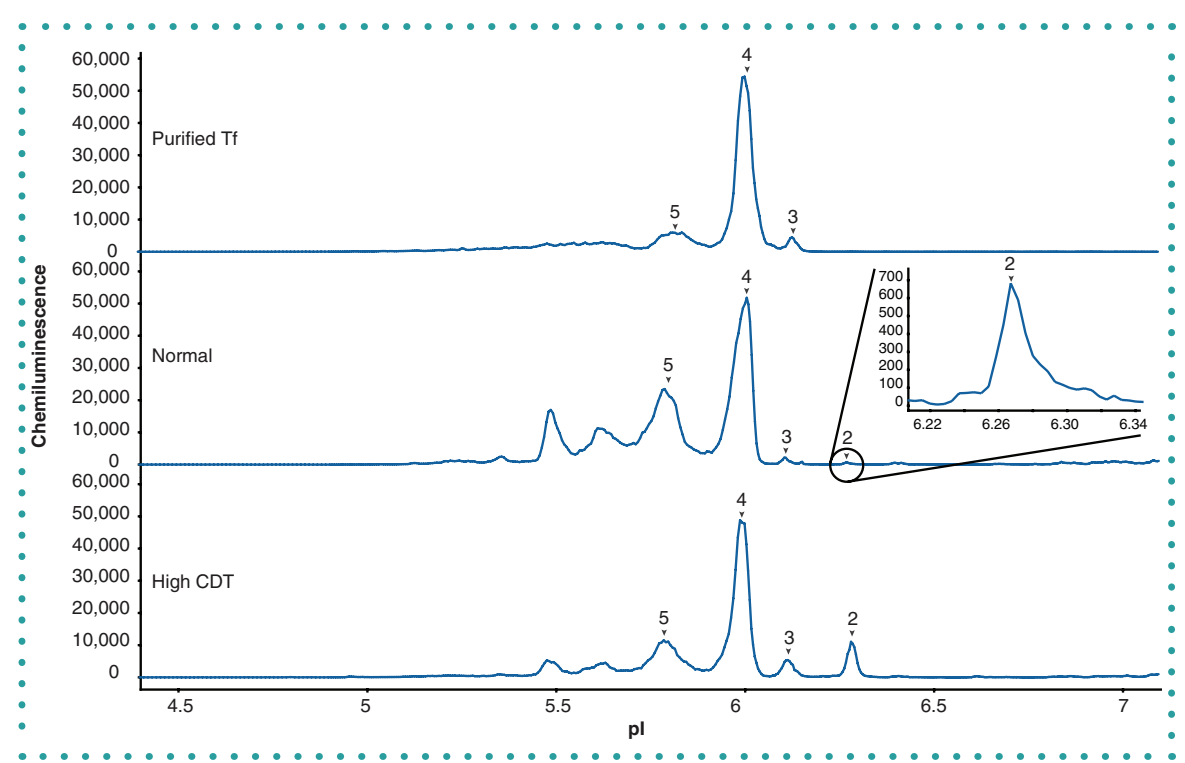

Figure 2. Electropherograms of purified transferrin and carbohydrate-deficient transferrin controls. The purified Tf sample concentration is $5 \mu \mathrm{g} / \mathrm{ml}$ and the CDT samples were diluted 600 -fold. Numbers above peaks correspond to Tf glycoforms; 5: pentasialo-Tf, 4: tetrasialo-Tf, 3: trisialo-Tf, 2: disialo-Tf. The inset shows a magnification of the disialo-Tf peak in the Normal Control. CDT: Carbohydrate-deficient transferrin; Tf: Transferrin. 


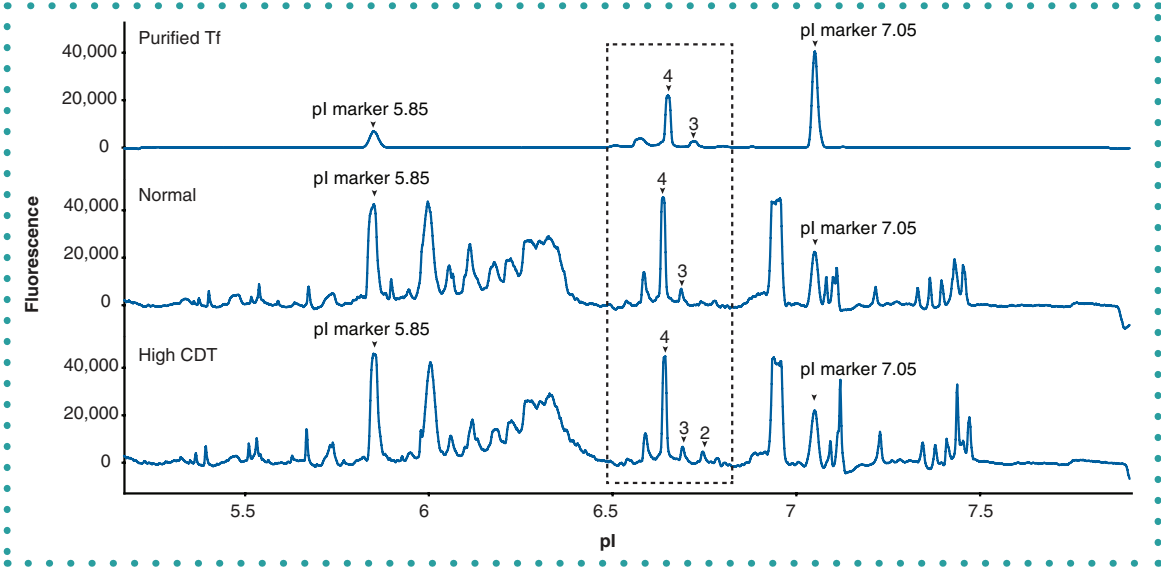

Figure 3. Analysis of purified transferrin and normal and high carbohydrate-deficient transferrin control samples by Maurice imaged capillary isoelectric focusing. Final concentrations: Purified Tf: $0.16 \mathrm{mg} / \mathrm{ml}$; CDT Controls: $1 / 25$ dilution. Numbers above peaks correspond to Tf glycoforms; 4: tetrasialo-Tf, 3: trisialo-Tf, 2: disialo-Tf.

CDT: Carbohydrate-deficient transferrin; Tf: Transferrin. to 96 samples (eight cycles) can be run in approximately $15 \mathrm{~h}$.

\section{Glycoform identification in samples by \\ iclEF peak patterns}

Following the workflow shown in Figure 1, we analyzed purified Tf and control serum samples containing a predetermined amount of CDT. These samples correspond to low (normal) and high (alcoholic) levels of CDT, called Normal Control and High CDT Control, respectively. The electropherograms resulting from this analysis are shown in Figure 2. According to manufacturer specifications for the Normal and High CDT Controls [15], the typical glycoforms include pentasialo-Tf $(\sim 18 \%)$, tetrasialo-Tf $(\sim 77 \%)$, trisialo-Tf ( $3 \%)$ and disialo-Tf $(\sim .9 \%)$ for the normal sample. For the High CDT Control sample, the typical

glycoforms include pentasialo-Tf ( 16\%), tetrasialo-Tf $(\sim 76 \%)$, trisialo-Tf $(\sim 3 \%)$ and disialo-Tf ( $5.1 \%)$. In our method, we were able to detect glycoform peaks above pentasialoTf (Figure 2). However, when considering the sum of only pentasialo-Tf through diasialo-Tf peaks for comparison, we measure pentasialo-Tf $(\sim 30 \%)$, tetrasialo-Tf( $68 \%)$, trisialo-Tf $(\sim 1 \%)$ and disialo-Tf $(\sim 0.5 \%)$ for the Normal Control; and pentasialo-Tf $(-23 \%)$, tetrasialo$\operatorname{Tf}(\sim 64 \%)$, trisialo-Tf $(\sim 5 \%)$ and disialo-Tf $(\sim 8 \%)$ for the High CDT Control. The differences in values described here are likely due to the different analytical techniques used; Tf peak profiles obtained by capillary zone electrophoresis and clEF are known to differ slightly and values between runs can also vary [1]. Nevertheless, in both the manufacturer specifications and our analysis, tetrasialo-Tf is the

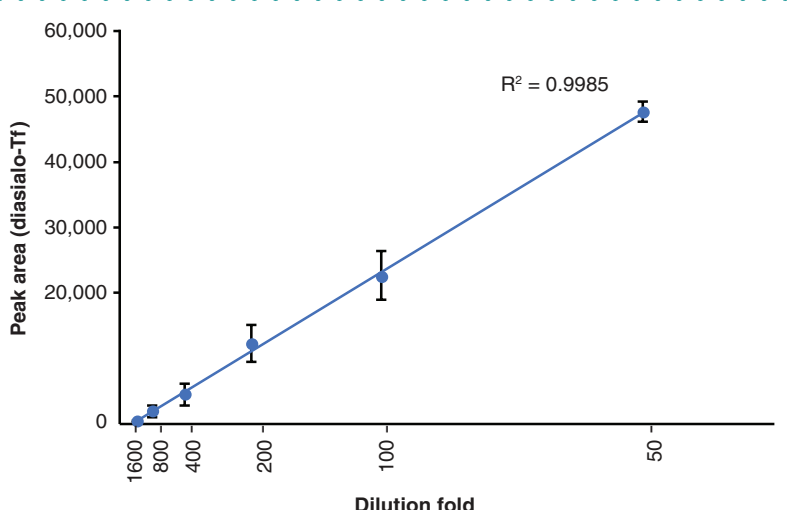

Figure 4. Peak area of carbohydrate-deficient transferrin (disialo-transferrin) versus sample dilution of a twofold serial dilution series of the normal control serum sample. Data are the averages of results from six replicates, and the error bars represent the standard errors of the means. Tf: Transferrin. predominant glycoform, as expected. Furthermore, disialo-Tf is the target analyte in IFCC-approved HPLC method for CDT [4], and its relative levels (diasialo-Tf to total Tf fraction percentage) are consistent with the method described here $(<2 \%$ for healthy individuals). It should be noted that the Tf in our samples has been treated with EDTA to remove bound iron. Because removing iron from Tf increases its apparent $\mathrm{pl}$, the pl values we measure here are higher than previous CIEF results of ferric Tf isoforms [16]. Instead, they align more closely with apoTf isoforms, as expected [16].

The results presented so far represent a novel detection method for CDT based on IEF followed seamlessly by immunodetection directly in the capillary. To understand how these results compare to traditional icIEF with native fluorescence detection, the same samples were analyzed by traditional iclEF, shown in Figure 3. As expected, a similar separation pattern for Tf was observed as previously published [11]. However, because this method detects all analytes in the sample at $280-\mathrm{nm}$ excitation and $320-450-\mathrm{nm}$ emission, the separation profile also includes other unrelated components that are present in serum. Therefore, combining icIEF with in-capillary immunodetection increases both specificity and sensitivity.

As in previously published icIEF methods, here Tf glycoforms are clearly separated and their apparent pl values can be precisely determined within $0.03 \mathrm{pH}$ units [11]. With such separation power and precision in apparent pl determination, minor peaks in the serum samples can be assigned to their corresponding glycoforms when the main tetrasialo peak is used as a reference. Similarly, CDT can be identified within the complete peak pattern of the sample without the need for a CDT-specific antibody, which can result in false-positives or false-negatives arising from uncommon $\mathrm{Tf}$ genetic isoforms or elevated levels of monosialo-Tf [17].

We examined the linear range of detection of CDT in human serum using the Normal Control sample. The sample was serially diluted twofold, from 50 -fold to 1600 -fold. The resulting data are shown in Figure 4. These results show linearity across a broad range $(\sim 30-$ fold $)$ of serum dilutions. From these results, the 600 -fold dilution was chosen for subsequent CDT analysis to provide enough material for CDT analysis while remaining 
in the determined linear range. A major advantage of this method is that only $15 \mu$ of the serum sample is needed, which is considerably less sample required by other methods that need $100 \mu \mathrm{l}$ of the serum sample [4].

Next, we sought to determine the LOQ of the method. As suggested by the International Conference on Harmonization of Technical Requirements for Registration of Pharmaceuticals for Human Use (ICH), the LOQ is determined 'based on the standard deviation of the response and the slope' [18]. Mathematically, the LOQ is defined as:

$L O Q=\frac{10 \sigma}{S}$

where $\sigma$ is the residual standard deviation of concentration calibration lines (standard deviation of $y$-intercepts of the lines), and $S$ is the slope of the calibration line. We used this formula to calculate the LOQ from three concentration calibration lines of the Normal Control sample. From this analysis, the LOQ of the method calculated to be at approximate the $1 / 1200$ dilution, which is well below the $1 / 600$ target dilution used in this study. Given that typical CDT levels are around $100 \mathrm{mg} / \mathrm{l}[16]$, this corresponds to as little as $83 \mu \mathrm{g} / \mathrm{l}$ detectable CDT.

Finally, we assessed assay reproducibility using the CDT controls. These results were obtained over 3 days, with six injections each day, totaling 18 injections. To visually demonstrate the reproducibility of this method, Figure 5 shows an overlay of three representative electropherograms performed over 3 days from each CDT control. Numerical values of the peak area percentages from all replicates are listed in Table 1, which reflects the intermediate precision of the method in determination of the Tf glycoforms. As expected, the results show significantly higher peak area percentage of the disialoglycoform in the High CDT Control sample compared with the Normal Control sample, with a mean increase of $6.5 \% \pm 0.8$, and this falls within with the specifications in the Sebia Normal and High CDT Control instructions for use, with a mean increase of $4.8 \pm 0.9$ (Sebia, GA, USA). Moreover, according to the specifications of the Normal Control Sample (Sebia), the mean CDT is $0.9 \% \pm 0.3$; therefore, the coefficient of variation is identical between our instrument and the manufacturer's specifications.

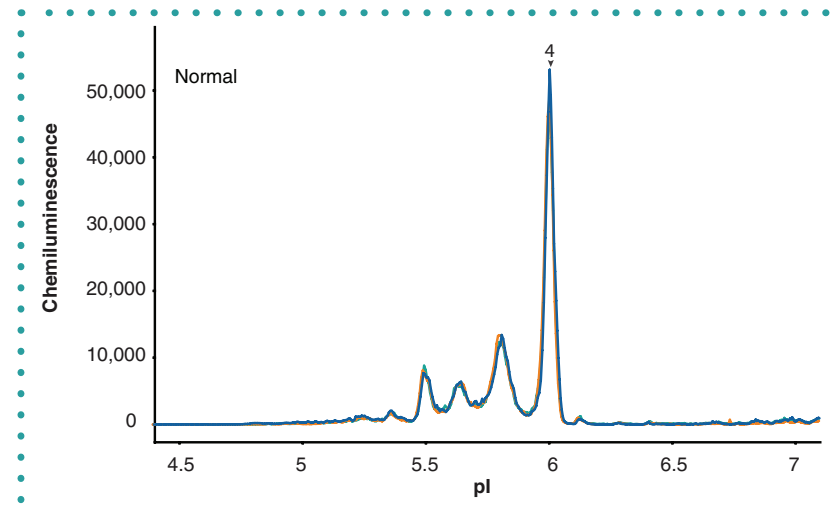

Figure 5. Representative

electropherograms

performed in triplicate

over the course of

3 days. Numbers above

peaks correspond to Tf

glycoforms; 4: tetrasialo-

Tf; 2: disialo-Tf.

CDT: Carbohydrate-

deficient transferrin;

Tf: Transferrin.

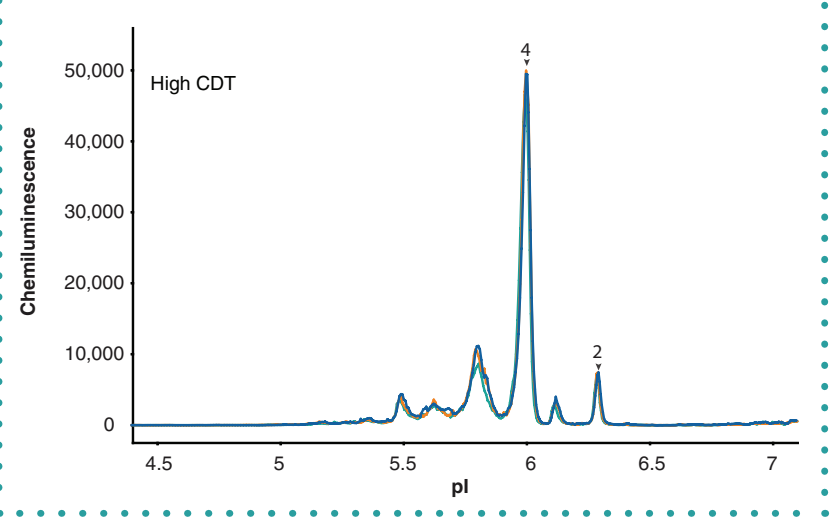

\section{CONCLUSION}

This work presents a new method of CDT analysis that employs icIEF followed seamlessly by in-capillary immunodetection on a single instrument (Peggy Sue from ProteinSimple). Like existing separationbased methods for CDT analysis that can identify genetic variants, including HPLC and $\mathrm{CE}$, this method separates and visualizes individual Tf isoforms. Because it has already been reported that cIEF is effective at identifying genetic variants of $\mathrm{Tf}[1]$, it is reasonable to anticipate that this method can do the same. However, unlike HPLC and CE methods, which detect any serological material with a specific UV or fluorescence wavelength, the method presented here utilizes a Tf-specific antibody for detection that takes place directly in the capillary following separation and capture of proteins to the capillary wall. The result is highly sensitive detection of individual Tf isoforms with minimal interference of non-Tf serological residents. This in turn reduces sample size and pretreatment steps. For example, the Sebia CDT assay requires $200 \mu \mathrm{l}$ of serum [15], and the IFCC-approved HPLC method requires $100 \mu$ of serum that must subjected to both iron saturation and delipidation [4]. By comparison, the described method requires $15 \mu$ of serum and only iron chelation pretreatment. The sensitivity of this method meets the standard set in the commercially available CDT immunodetection assay [7] with the added advantage of providing accurate apparent pl values of each isoform when standards are included. Following minor pretreatment, serum samples are diluted directly in IEF sample buffer, and a batch of up to 96 samples can be analyzed overnight in a fully automated fashion. This method eliminated the need for immunopurification of $\mathrm{Tf}$ from serum samples found in other IEF methods [8].

A potential limitation of the commercially available immunoassay for CDT (N Latex CDT assay) is that it relies on an antibody that also detects monosialo-Tf, which is not considered CDT [7]. Instead, elevated monosialo-Tf is typically associated with elevated levels of trisialo-Tf and is not believed to be related to chronic alcohol abuse. Additionally, trisialo-Tf is not detected by the N Latex CDT assay [7], so these conditions would go unnoticed. By contrast, this IEF-based method provides the full-charge separation profile of the sample, and trisialo-Tf can be easily distinguished from CDT glycoforms, and, therefore, elevated trisialo-Tf levels can be identified. Finally, this method measured a significant increase $(6.5 \% \pm 0.8)$ in the diasialo-glycoform level in the High CDT Control sample compared with the Normal Control sample that falls 


\section{Table 1. Percent peak area of tetrasialo- and disialo-glycoforms in carbohydrate-deficient transferrin controls.}

\begin{tabular}{|c|c|c|c|c|}
\hline & & CDT (\%) & & DT (\%) \\
\hline & Tetrasialo & Disialo & Tetrasialo & Disialo \\
\hline & 45.8 & 0.3 & 52.5 & 6.4 \\
\hline & 48.4 & 0.2 & 55.4 & 5.9 \\
\hline & 48.7 & 0.5 & 53.9 & 5.3 \\
\hline & 48.9 & 0.3 & 58.2 & 6.7 \\
\hline & 47.7 & 0.4 & 57.3 & 6.3 \\
\hline & 47.4 & 0.4 & 55.0 & 6.9 \\
\hline & 48.4 & 0.3 & 45.8 & 6.2 \\
\hline & 41.2 & 0.3 & 47.5 & 6.1 \\
\hline & 47.1 & 0.3 & 49.1 & 5.8 \\
\hline & 45.9 & 0.3 & 49.3 & 8.5 \\
\hline & 47.0 & 0.2 & 46.3 & 7.8 \\
\hline & 49.9 & 0.3 & 47.0 & 7.0 \\
\hline & 41.1 & 0.2 & 48.0 & 7.7 \\
\hline & 44.2 & 0.3 & 48.4 & 7.2 \\
\hline & 48.9 & 0.3 & 51.9 & 6.9 \\
\hline & 43.5 & 0.4 & 49.9 & 6.9 \\
\hline & 43.4 & 0.2 & 44.9 & 7.5 \\
\hline & 46.5 & 0.5 & 46.1 & 7.0 \\
\hline Mean & 46.3 & 0.3 & 50.4 & 6.8 \\
\hline SD & 2.6 & 0.1 & 4.0 & 0.8 \\
\hline Range & $38.5-54.1$ & $0-0.6$ & $38.4-62.4$ & $4.4-9.2$ \\
\hline
\end{tabular}

within the expected range of $4.8 \% \pm 0.9$. At an estimated cost of approximately $\$ 6-7$ per sample [19], this method may be an affordable alternative to the reported average cost of CDT analysis (\$30/assay) [20]. Collectively, these results indicate that this method is a valuable tool in CDT analysis.

\section{FUTURE PERSPECTIVE}

Different techniques for Tf/CDT analysis currently exist based on protein separation or immunoassay. This study provides a foundation for exploring new alternatives to identifying and characterizing Tf/CDT by combining the power of charge isoform separation and sensitivity of an immunoassay on a single platform, Simple Western. Further investigation of Tf/CDT by Simple Western Charge may include the use of antibodies specific to Tf glycoforms or genetic variants, and the application of the assay to atypical samples and large sample sets.

\section{AUTHOR CONTRIBUTIONS}

$\mathrm{JWu}$ and CD Heger planned the experiments. $\mathrm{J}$ Wu ran the experiments. J Wu, CD Heger and $\mathrm{A}$ Boge analyzed the data. $\mathrm{J} \mathrm{Wu}, \mathrm{CH}$ Haitjema and CD Heger wrote the manuscript.

\section{FINANCIAL \& COMPETING INTERESTS DISCLOSURE}

J Wu, CD Heger, CH Haitjema and A Boge are employees of and have stock ownership in Bio-Techne. The authors have no other relevant affiliations or financial involvement with any organization or entity with a financial interest in or financial conflict with the subject matter or materials discussed in the manuscript apart from those disclosed.

No writing assistance was utilized in the production of this manuscript.

\section{OPEN ACCESS}

This work is licensed under the AttributionNonCommercial-NoDerivatives 4.0 Unported License. To view a copy of this license, visit http://creativecommons.org/licenses/ by-nc-nd/4.0/

\section{REFERENCES}

1. Caslavska J, Thormann W. Monitoring of transferrin isoforms in biological samples by capillary electrophoresis. J. Sep. Sci. 41(1), 303-322 (2018).

2. Bortolotti F, Sorio D, Bertaso A, Tagliaro F. Analytical and diagnostic aspects of carbohydrate deficient transferrin (CDT): a critical review over years 2007-2017. J. Pharm. Biomed. Anal. 147, 2-12 (2018).

3. Yoo G, Kim J, Yoon KJ, Lee JH. The characteristics of transferrin variants by carbohydrate-deficient transferrin tests using capillary zone electrophoresis. J. Clin. Lab. Anal. 32(7), 1-4 (2018).

4. Schellenberg F, Wielders J, Anton R et al. IFCC approved HPLC reference measurement procedure for the alcohol consumption biomarker carbohydrate-deficient transferrin (CDT): its validation and use. Clin. Chim. Acta 465, 91-100 (2017).

5. Musile G, De Palo EF, Savchuk SA et al. A novel low-cost approach for the semi-quantitative analysis of carbohydrate-deficient transferrin (CDT) based on fluorescence resonance energy transfer (FRET). Clin. Chim. Acta 495 556-561 (2019).

6. Nicotra S, Sorio D, Filippi G et al. Terbium chelation, a specific fluorescent tagging of human transferrin. Optimization of conditions in view of its application to the HPLC analysis of carbohydrate-deficient transferrin (CDT). Anal. Bioanal. Chem. 409(28), 6605-6612 (2017).

7. Delanghe JR, Helander A, Wielders JPM et al. Development and multicenter evaluation of the N Latex CDT direct immunonephelometric assay for serum carbohydrate-deficient transferrin. Clin. Chem. 53(6), 1115-1121 (2007).

8. Caslavska J, Lanz C, Burda P, Tobler M, Thormann W. Analysis of genetic variants of transferrin in human serum after desialylation by capillary zone electrophoresis and capillary isoelectric focusing. J. Sep. Sci. 40(11), 2488-2497 (2017).

9. Wu J, Pawliszyn J. A capillary cartridge with an online desalting device that allows fast sampling for capillary isoelectric focusing. Anal. Chem. 67(13), 2010-2014 (2005).

10. Salas-Solano O, Kennel B, Park SS et al. Robustness of iCIEF methodology for the analysis of monoclonal antibodies: an interlaboratory study. J. Sep. Sci. 35(22), 3124-3129 (2012).

11. Wu J, Huang T. Peak identification in capillary isoelectric focusing using the concept of relative peak position as determined by two isoelectric point markers. Electrophoresis 27(18), 3584-3590 (2006).

12. Gentalen E, White T, Proctor J. Peggy ${ }^{\mathrm{Tm}}$ : size- or chargebased western blotting at the push of a button. Nat. Methods 10, i-ii (2013).

13. Harris VM. Protein detection by simple western ${ }^{\text {TM }}$ analysis. Western Blotting. Methods Mol. Biol. 1312, 465-468 (2015).

14. Markely LRA, Cheung L, Choi YJ et al. A high-throughput capillary isoelectric focusing immunoassay for fingerprinting protein sialylation. Biotechnol. Prog. 32(1), 235-241 (2016).

15. Sebia. CDT capillarys. http://www.sebia.com/en-EN/produits/capillarys-cdt

16. Arndt T. Carbohydrate-deficient transferrin as a marker of chronic alcohol abuse: a critical review of preanalysis, analysis, and interpretation. Clin. Chem. 47(1), 13-27 (2001).

17. Helander A, Eriksson G, Stibler H, Jeppsson JO. Interference of transferrin isoform types with carbohydrate-deficient transferrin quantification in the identification of alcohol abuse. Clin. Chem. 47(7), 1225-1233 (2001).

18. ICH-Expert-Working-Group. ICH Topic Q2 (R1) validation of analytical procedures: text and methodology (2005). https:// database.ich.org/sites/default/files/Q2_R1_Guideline.pdf

19. ProteinSimple. XDR Charge Separation Master Kit for Peggy Sue, Peggy or NanoPro 1000. shop.proteinsimple.com/ nanopro-1000-xdr-master-kit.html

20. Spiegel D, Dhadwal N, Gill F. 'I'm sober, Doctor, really': best biomarkers for underreported alcohol use. Curr. Psychiatr. $7(9), 15-27$ (2008) 